\title{
A Neoteric Three-Dimensional Geometry-Based Stochastic Model for Massive MI MO Fading Channels in Subway Tunnels
}

\author{
Yukang Jiang ${ }^{1}$, Aihuang Guo ${ }^{1,2 *}$, Jinbai $\mathrm{Zou}^{1,3}$ and $\mathrm{Bo} \mathrm{Ai}^{2}$ \\ ${ }^{1}$ School of Electronics \& Information Engineering, Tongji University, \\ Shanghai 201804, China \\ [e-mail: jiang_yukang@163.com; tjgah@tongji.edu.cn] \\ ${ }^{2}$ State Key Laboratory of Rail Traffic Control and Safety, Beijing Jiaotong University, \\ Beijing 100044, China \\ [e-mail: boai@bjtu.edu.cn] \\ ${ }^{3}$ School of Railway Transportation, Shanghai Institute of Technology, \\ Shanghai 201400, China \\ [e-mail: zoujb@sit.edu.cn] \\ *Corresponding author: Aihuang Guo
}

Received March 19, 2018; revised July 16, 2018; accepted December 28, 2018;

published June 30, 2019

\begin{abstract}
Wireless mobile communication systems in subway tunnels have been widely researched these years, due to increased demand for the communication applications. As a result, an accurate model is essential to effectively evaluate the communication system performance. Thus, a neoteric three-dimensional (3D) geometry-based stochastic model (GBSM) is proposed for the massive multiple-input multiple-output (MIMO) fading channels in tunnel environment. Furthermore, the statistical properties of the channel such as space-time correlation, amplitude and phase probability density are analyzed and compared with those of the traditional two-dimensional (2D) model by numerical simulations. Finally, the ergodic capacity is investigated based on the proposed model. Numerical results show that the proposed model can describe the channel in tunnels more practically.
\end{abstract}

Keywords: Wireless communication, massive MIMO system, 3D GBSM, statistical properties, channel capacity, tunnel environment

This work is supported by the State Key Laboratory of Rail Traffic Control and Safety (No. 2017k013). 


\section{Introduction}

$\mathbf{I}_{\mathrm{N}}$ recent years, the rapid development of urban rail transit has resulted in increased demand for communication applications in subway. As a result, the wireless mobile communication systems in tunnels is extensively studied. Different from the traditional mobile communication channels on the ground, those in tunnels are confined in a limited space. Moreover, the massive multiple-input multiple-output (MIMO) technologies [1-2], where multiple antennas are eployed at both the transmitter (Tx) and receiver (Rx) [3], have been receving more and more attention in tunnel communication systems. Multiple antennas are helpful to bring huge improvements in throughput and radiated energy efficiency by focusing energy into small regions of space. Other benefits of massive MIMO include extensive use of low-power components, reduced latency and robustness against intentional jamming [4].

In order to design an applicative communication system in tunnel environment and evaluate the system performance, an accurate channel model and a detailed knowledge of its statistical properties are indispensable. There are already many useful models adapted in different environments, such as ray-tracing models [5-6], geometry-based deterministic models (GBDMs) [7], correlation-based stochastic models (CBSMs) [8-9] and geometry-based stochastic models (GBSMs) [10-11]. Because of the research convenience for the statistical characteristics and capacity, GBSMs are most widely used. In GBSMs, the propagation characteristics of radio waves mainly depend on randomly distributed scatterers in different environment [12]. Hence, GBSMs can be adapted to different communication scenarios by modifying the random distribution of scatter clusters and the shape of the scatter area.

There are already many available GBSMs for different communication environments. A two-dimensional (2D) model with "one-ring" or "two-ring" is proposed in [13-14] for the narrowband and wideband MIMO channels. In [15], Zheng and Nie proposed a 2D scattering model and analyzed the corresponding statistical properties. However, 2D models assume that waves travel only in the horizontal plane and neglect the vertical plane so that they cannot describe the practical three-dimensional (3D) space accurately. Therefore, in [16], the authors put forward a 3D single-ellipsoids GBSM for both outdoor and indoor environments. In [17], the 3D two-cylinder GBSM was proposed for narrowband MIMO mobile-to-mobile system. In [18], authors improved the model in [17] and developed a novel 3D one-elliptic-cylinder with two-sphere GBSM for non-isotropic MIMO vehicle-to-vehicle channels. Also, a two-sphere model [19] was provided to describe closed indoor scenarios and a two-cylinder model [12] was proposed to describe high-speed scenarios.

The aforementioned GBSMs have not considered the train's motion and the limited space jointly, thus, they cannot be applied directly for the particular channels in tunnels. As a result, we propose a novel theoretical 3D GBSM for the non-isotropic massive MIMO channels adapted to the tunnel environment. The proposed GBSM is combined with a horizontal cylinder model and a sphere model which is inscribed with the cylinder. Line-of-sight (LOS), single-reflection (SR) and double-reflection (DR) components are considered in this model. Based on the proposed model, some relevant comprehensive statistics are derived, i.e., the space-time correlation function (STCF), amplitude and phase probability density functions (PDFs). Then, the impact of factors on the space-time correlation are investigated and the channel capacity is analyzed. 
The remainding of this paper is organized as follows. Section 2 proposes a new 3D GBSM for massive MIMO channels in subway tunnels. In section 3, the corresponding statistical properties and channel capacity are derived. The statistics and capacity are thoroughly investigated by simulations and analyzed in section 4 . Finally, the corresponding research is summarized in section 5 .

\section{A Neoteric 3D Massive MIMO GBSM for Channels in Subway Tunnels}

A massive MIMO wireless mobile communication system in tunnels is considered in this paper. It is supposed that the Tx and Rx are equipped with $M_{T}$ and $M_{R}$ number of antenna elements, respectively. Tx is stationary however, Rx is mobile. Fig. 1 illustrates our proposed GBSM with a horizontal cylinder model and a sphere model inscribed with the cylinder. The horizontal cylinder of radius $R_{C}$ is used to describe the tunnel wall and the sphere of radius $R_{S}\left(R_{S}=R_{C}\right)$ is used to mimic the moving trains. In Fig. 1, the antenna element at the Tx is numbered as $p=1,2, \ldots, M_{T}$ and the one at the $\mathrm{Rx}$ is numbered as $q=1,2, \ldots, M_{R}$, respectively. The antennas at both Tx and Rx can be extended to arbitrary numbers, and the antenna arrays can be configured with any shapes. Moreover, the communication channel is depicted with the composition of the LOS, SR and DR components. The SR radio waves are incident on the cylinder and the DR ones are incident on both the sphere and cylinder. Other bounced paths are ignored because they reflect similar channel characteristics and the signal power is reduced sharply after multiple reflections [12]. In addition, there are $N_{1}$ omnidirectional effective scatterers distributed on the sphere model and $N_{2}$ scatters on the cylinder one. The $n_{1}$ th $\left(n_{1}=1,2, \ldots, N_{1}\right)$ receive scatterer is signified by $S^{\left(n_{1}\right)}$ and the $n_{2}$ th $\left(n_{2}=1,2, \ldots, N_{2}\right)$ scatterer is signified by $S^{\left(n_{2}\right)}$. The parameters in Fig. 1 are defined in Table 1. Considering the actual environment, reasonable assumptions $D>>R_{S}$ and $\max \left\{\delta_{T}, \delta_{R}\right\}<<R_{S}$ are used in the proposed GBSM.

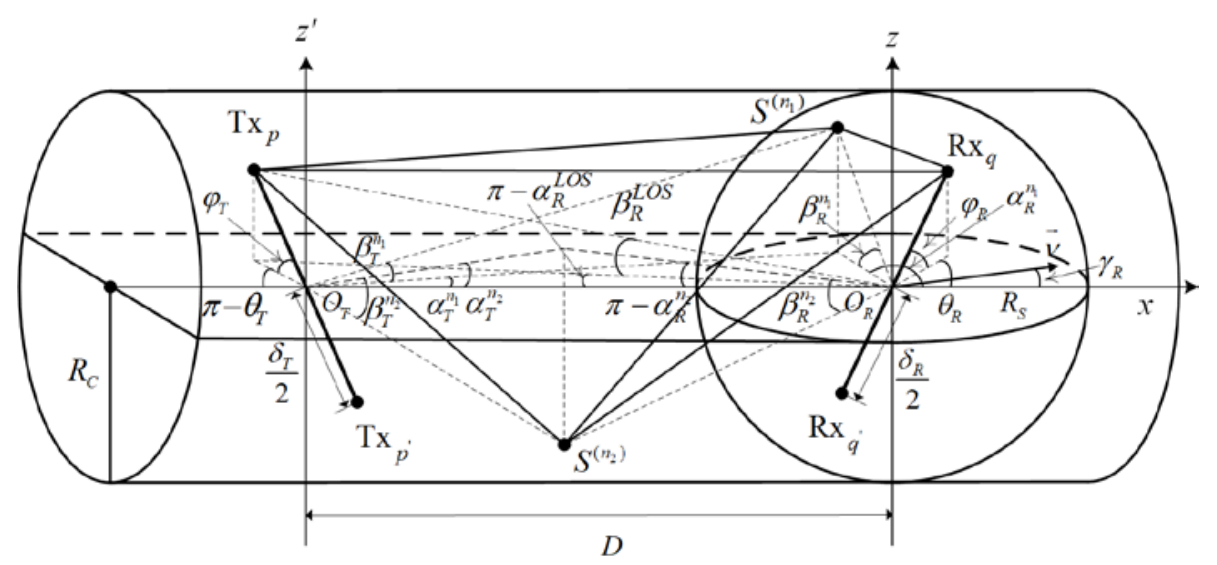

Fig. 1. The 3D massive MIMO GBSM combining a horizontal cylinder model and a sphere one 
Table 1. Definitions of symbols used in Fig. 1

\begin{tabular}{|c|c|}
\hline Symbols & Definitions \\
\hline$D$ & the distance between the centers of the Tx and Rx \\
\hline$O_{T}, O_{R}$ & the central position of the $\mathrm{Tx}$ and $\mathrm{Rx}$ \\
\hline$R_{C}, R_{S}$ & the radius of the sphere and cylinder models, respectively \\
\hline$\delta_{T}, \delta_{R}$ & $\begin{array}{l}\text { the spacing between antenna elements of the Tx and } \mathrm{Rx} \text {, } \\
\text { respectively }\end{array}$ \\
\hline$\theta_{T}, \theta_{R}$ & $\begin{array}{l}\text { the azimuth angle of the Tx and Rx antenna array relative to } \\
\text { the } x \text {-axis, respectively }\end{array}$ \\
\hline$\varphi_{T}, \varphi_{R}$ & $\begin{array}{l}\text { the elevation angle of the Tx and Rx antenna array relative to } \\
\text { the } x-y \text { plane, respectively }\end{array}$ \\
\hline$\gamma_{R}$ & the subway running direction relative to the $x$-axis \\
\hline$v$ & $\begin{array}{l}\text { the subway running velocities } \\
\end{array}$ \\
\hline$\alpha_{R}^{\mathrm{LOS}}, \beta_{R}^{\mathrm{LOS}}$ & the azimuth and elevation angles of arrival of the LOS path \\
\hline$\alpha_{T}^{n_{i}}, \beta_{T}^{n_{i}}(i=1,2)$ & $\begin{array}{l}\text { the azimuth and elevation angels of departure of the waves } \\
\text { impinging on the scatters } S^{\left(n_{i}\right)}\end{array}$ \\
\hline$\alpha_{R}^{n_{i}}, \beta_{R}^{n_{i}}(i=1,2)$ & $\begin{array}{l}\text { the azimuth and elevation angles of arrival of the waves } \\
\text { reflecting from the scatters } S^{\left(n_{i}\right)}\end{array}$ \\
\hline
\end{tabular}

The 3D massive MIMO channel in tunnels can be described by $\mathbf{H}(t)=\left[h_{p q}(t)\right]_{M_{T} \times M_{R}}$ matrix . The element $h_{p q}(t)$ in the matrix denotes the received complex fading envelope of the link between the $p$ th transmitting antenna and the $q$ th receiving antenna at the carrier frequency of $f_{c} \cdot h_{p q}(t)$ is a superposition of the LOS, SR and DR components, which can be expressed as [18]

$$
h_{p q}(t)=h_{p q}^{\mathrm{LOS}}(t)+\sum_{i=1}^{I} h_{p q}^{\mathrm{SR}_{i}}(t)+h_{p q}^{\mathrm{DR}}(t)
$$

where $I=2$, which means there are two subcomponents for SR rays. Furthermore, $\mathrm{SR}_{1}$ appears on the sphere and $\mathrm{SR}_{2}$ appears on the cylinder. The complex fading envelope of each part can be respectively written as

$$
\begin{aligned}
h_{p q}^{\mathrm{LOS}}(t) & =\sqrt{\frac{K}{K+1}} \exp \left(-\frac{\mathrm{j} 2 \pi}{\lambda} \varepsilon_{p q}\right) \cdot \exp \left[\mathrm{j} 2 \pi f_{\max } t \cos \left(\alpha_{R}^{\mathrm{LOS}}-\gamma_{R}\right) \cos \beta_{R}^{\mathrm{LOS}}\right] \\
h_{p q}^{\mathrm{SR}}(t)= & \sqrt{\frac{\eta_{\mathrm{SR}_{i}}}{K+1}} \lim _{N_{i} \rightarrow \infty} \sum_{n_{i}=1}^{N_{i}} \frac{1}{\sqrt{N_{i}}} \cdot \exp \left\{\mathrm{j}\left[\psi_{n_{i}}-\frac{2 \pi}{\lambda}\left(\varepsilon_{p n_{i}}+\varepsilon_{n_{i} q}\right)\right]\right\} \\
& \cdot \exp \left\{\mathrm{j}\left[2 \pi f_{\max } t \cos \left(\alpha_{R}^{n_{i}}-\gamma_{R}\right) \cos \beta_{R}^{n_{i}}\right]\right\} \\
h_{p q}^{\mathrm{DR}}(t)= & \sqrt{\frac{\eta_{\mathrm{DR}}}{K+1}} \lim _{N_{1}, N_{2} \rightarrow \infty} \sum_{n_{1}, n_{2}=1}^{N_{1}, N_{2}} \frac{1}{\sqrt{N_{1} N_{2}}} \cdot \exp \left\{\mathrm{j}\left[\psi_{n_{1}, n_{2}}-\frac{2 \pi}{\lambda}\left(\varepsilon_{p n_{1}}+\varepsilon_{n_{1} n_{2}}+\varepsilon_{n_{2} q}\right)\right]\right\} \\
& \cdot \exp \left\{\mathrm{j}\left[2 \pi f_{\max _{1}} t \cos \left(\alpha_{R}^{n_{2}}-\gamma_{R}\right) \cos \beta_{R}^{n_{2}}\right]\right\}
\end{aligned}
$$


where $K$ is the Rician factor, $\lambda$ is the wavelength of light, $N_{i}$ is the number of effective scatterers and $f_{\max }$ is the maximum Doppler frequency at the Rx. Here, $\psi_{n_{i}} 、 \psi_{n_{1}, n_{2}}$ are independent and identically distributed (i.i.d) random variables with uniform distributions between the range $[-\pi, \pi), \eta_{\mathrm{SR}_{i}}$ and $\eta_{\mathrm{DR}}$ are power-related coefficients satisfying $\sum_{i=1}^{I} \eta_{\mathrm{SR}_{i}}+\eta_{\mathrm{DR}}=1 . \varepsilon_{p q}, \varepsilon_{p n_{i}}, \varepsilon_{n_{i} q}$ and $\varepsilon_{n_{1} n_{2}}$ denote $d\left(\operatorname{Tx}_{p}, \mathrm{Rx}_{q}\right), d\left(\operatorname{Tx}_{p}, S^{\left(n_{i}\right)}\right), d\left(S^{\left(n_{i}\right)}, \mathrm{Rx}_{q}\right)$ and $d\left(S^{\left(n_{1}\right)}, S^{\left(n_{2}\right)}\right)(d(\mathrm{X}, \mathrm{Y})$ denotes the $\mathrm{X}$-to-Y distance, $\mathrm{X}$ and $\mathrm{Y}$ are position points), respectively, which can be expressed as follows:

$$
\begin{aligned}
\varepsilon_{p q}= & {\left[\left(D-\frac{\delta_{T}}{2} \cos \varphi_{T} \cos \theta_{T}+\frac{\delta_{R}}{2} \cos \varphi_{R} \cos \theta_{R}\right)^{2}\right.} \\
& \left.+\left(\frac{\delta_{T}}{2} \cos \varphi_{T} \sin \theta_{T}-\frac{\delta_{R}}{2} \cos \varphi_{R} \sin \theta_{R}\right)^{2}+\left(\frac{\delta_{T}}{2} \sin \varphi_{T}-\frac{\delta_{R}}{2} \sin \varphi_{R}\right)^{2}\right]^{1 / 2} \\
\varepsilon_{p n_{1}}= & {\left[\left(\frac{\delta_{T}}{2} \cos \varphi_{T} \sin \theta_{T}-R_{S} \cos \beta_{R}^{n_{1}} \sin \alpha_{R}^{n_{1}}\right)^{2}\right.} \\
& \left.+\left(D-\frac{\delta_{T}}{2} \cos \varphi_{T} \cos \theta_{T}+R_{S} \cos \beta_{R}^{n_{1}} \cos \alpha_{R}^{n_{1}}\right)^{2}+\left(\frac{\delta_{T}}{2} \sin \varphi_{T}-R_{S} \sin \beta_{R}^{n_{1}}\right)^{2}\right]^{1 / 2} \\
\varepsilon_{n_{1} q}= & {\left[\left(\frac{\delta_{R}}{2} \cos \varphi_{R} \cos \theta_{R}-R_{S} \cos \beta_{R}^{n_{1}} \cos \alpha_{R}^{n_{1}}\right)^{2}\right.} \\
& \left.+\left(\frac{\delta_{R}}{2} \cos \varphi_{R} \sin \theta_{R}-R_{S} \cos \beta_{R}^{n_{1}} \sin \alpha_{R}^{n_{1}}\right)^{2}+\left(\frac{\delta_{R}}{2} \sin \varphi_{R}-R_{S} \sin \beta_{R}^{n_{1}}\right)^{2}\right]^{1 / 2} \\
\varepsilon_{p n_{2}}= & {\left[\left(\xi_{T n_{2}} \cos \beta_{T}^{n_{2}} \cos \alpha_{T}^{n_{2}}-\frac{\delta_{T}}{2} \cos \varphi_{T} \cos \theta_{T}\right)^{2}\right.} \\
& \left.+\left(\frac{\delta_{T}}{2} \cos \varphi_{T} \sin \theta_{T}-\xi_{T n_{2}} \cos \beta_{T}^{n_{2}} \sin \alpha_{T}^{n_{2}}\right)^{2}+\left(\frac{\delta_{T}}{2} \sin \varphi_{T}+\xi_{T n_{2}} \sin \beta_{T}^{n_{2}}\right)^{2}\right]^{1 / 2} \\
& \left.+\left(R_{S} \cos \beta_{R}^{n_{1}} \sin \alpha_{R}^{n_{1}}-\xi_{R n_{2}} \cos \beta_{R}^{n_{2}} \sin \alpha_{R}^{n_{2}}\right)^{2}\right]^{1 / 2} \\
\varepsilon_{n_{1} n_{2}}= & {\left[\left(R_{S} \sin \beta_{R}^{n_{1}}+\xi_{R n_{2}} \sin \beta_{R}^{n_{2}}\right)^{2}+\left(R_{S} \cos \beta_{R}^{n_{1}} \cos \alpha_{n_{1}}^{n_{1}}-\xi_{R n_{2}} \cos \beta_{R}^{n_{2}} \cos \alpha_{R}^{n_{2}}\right)^{2}\right.} \\
& \left.\left.+\left(\frac{\delta_{R}}{2} \cos \varphi_{R} \cos \theta_{R}-\xi_{R n_{2}} \cos \beta_{R}^{n_{2}} \cos \alpha_{R}^{n_{2}}\right)^{2} \sin \theta_{R}-\xi_{R n_{2}} \cos \beta_{R}^{n_{2}} \sin \alpha_{R}^{n_{2}}\right)^{2}+\left(\frac{\delta_{R}}{2} \sin \varphi_{R}+\xi_{R n_{2}} \sin \beta_{R}^{n_{2}}\right)^{2}\right]^{1 / 2}
\end{aligned}
$$

where $\xi_{T n_{2}}=R_{C} / \sin \left[\arccos \left(\cos \beta_{T}^{n_{2}} \cos \alpha_{T}^{n_{2}}\right)\right]$ and $\xi_{R n_{2}}=R_{C} / \sin \left[\arccos \left(\cos \beta_{R}^{n_{2}} \cos \left(\pi-\alpha_{R}^{n_{2}}\right)\right)\right]$.

Note that the determined scatterer distribution needs to be applied in this particular model for the subsequent study of relevant statistical properties. In this paper, to consider the effect of 
the azimuth and elevation angles jointly, von Mises-Fisher (VMF) distribution is used to characterize the effective scatterer distribution, whose PDF is defined as [20]

$$
f(\alpha, \beta)=\frac{k \cos \beta}{4 \pi \sinh k} \cdot \exp \left[k\left(\cos \beta \cos \beta_{0} \cos \left(\alpha-\alpha_{0}\right)+\sin \beta \sin \beta_{0}\right)\right]
$$

where $\alpha \in[-\pi, \pi), \beta \in[-\pi / 2, \pi / 2), \alpha_{0} \in[-\pi, \pi)$ and $\beta_{0} \in[-\pi / 2, \pi / 2)$ represent the average values of azimuth and elevation angles, respectively, and $k$ is an environmental coefficient which controls the scatterer spread around the mean value.

\section{Statistical Properties and Channel Capacity of the 3D Massive MIMO GBSM}

The massive MIMO system is capable of making full use of space resources and can achieve the spatial parallel transmission of radio waves. Furthermore, the link reliability and channel capacity can be improved significantly by using space-time joint processing method [21]. In order to evaluate the performance of the 3D GBSM in tunnels more effectively and associate the effective scatterers in the propagation environment with the multiple antennas' performance, the statistical properties, such as STCF and PDFs, and the capacity need to be analyzed.

\subsection{STCF}

Both the spatial and temporal correlations exist in any fading channels. The spatial correlation describes the cross-correlation properties between different antenna elements while the temporal one depicts the auto-correlation properties of a single receiving antenna element at different time instances. The STCF can be used to describe the impact of both spatial and temporal correlations on wireless communication systems.

Under the wide sense stationary uncorrelated scattering condition [22], the normalized STCF between any two sub-channels can be derived from the complex fading envelope showed in (1) and written as [23]

$$
\begin{aligned}
\rho_{p q, p^{\prime} q^{\prime}}\left(\delta_{T}, \delta_{R}, \tau\right) & =\frac{\mathbf{E}\left[h_{p q}(t) h_{p^{\prime} q^{\prime}}^{*}(t+\tau)\right]}{\sqrt{\mathbf{E}\left[\left|h_{p q}(t)\right|^{2}\right] \cdot \mathbf{E}\left[\left|h_{p^{\prime} q^{\prime}}(t)\right|^{2}\right]}} \\
& =\rho_{p q, p^{\prime} q^{\prime}}^{\mathrm{LOS}}\left(\delta_{T}, \delta_{R}, \tau\right)^{+} \sum_{i=1}^{I} \rho_{p q^{\prime} p^{\prime} q^{\prime}}^{\mathrm{SR}_{i}}\left(\delta_{T}, \delta_{R}, \tau\right)+\rho_{p q, p^{\prime} q^{\prime}}^{\mathrm{DR}}\left(\delta_{T}, \delta_{R}, \tau\right)
\end{aligned}
$$

where $\mathbf{E}[\cdot]$ denotes the statistical expectation operator and $(\cdot)^{*}$ is the complex conjugate operation, $p, p^{\prime} \in\left(1,2, \ldots, M_{T}\right)$ and $q, q^{\prime} \in\left(1,2, \ldots, M_{R}\right)$.

Substituting (2)-(4) into (12) and using the accordant VMF scattering distribution at the same time, the STCF of LOS, SR and DR components is derived next.

\section{1) The STCF of LOS component}

The STCF of LOS component is expressed as

$$
\rho_{p q, p^{\prime} q^{\prime}}^{\mathrm{LOS}}\left(\delta_{T}, \delta_{R}, \tau\right)=K \cdot \exp \left(\frac{\mathrm{j} 2 \pi}{\lambda} \cdot D_{\mathrm{LOS}}\right) \cdot \exp \left[\mathrm{j} 2 \pi \tau f_{\max } \cos \left(\alpha_{R}^{\mathrm{LOS}}-\gamma_{R}\right) \cos \beta_{R}^{\mathrm{LOS}}\right]
$$

where $D_{\mathrm{LOS}}=2 D \cos \varphi_{R} \cos \theta_{R}$. Furthermore, considering $D>>R_{S}$, we have the following 
approximations $\alpha_{R}^{\mathrm{LOS}} \approx \pi$ and $\alpha_{T}^{\mathrm{LOS}} \approx \beta_{T}^{\mathrm{LOS}} \approx \beta_{R}^{\mathrm{LOS}} \approx 0$. Therefore, Eq. (13) can be simplified as

$$
\rho_{p q, p^{\prime} q^{\prime}}^{\mathrm{LOS}}\left(\delta_{T}, \delta_{R}, \tau\right)=K \cdot \exp \left(\frac{\mathrm{j} 2 \pi}{\lambda} D_{\mathrm{LOS}}\right) \cdot \exp \left(-\mathrm{j} 2 \pi \tau f_{\max } \cos \gamma_{R}\right)
$$

\section{2) The STCF of SR component}

The STCF of SR component is expressed as

$$
\begin{aligned}
\rho_{p q, p^{\prime} q^{\prime}}^{\mathrm{SR}_{i}}\left(\delta_{T}, \delta_{R}, \tau\right)= & \eta_{\mathrm{SR}_{i}} \int_{-\pi}^{\pi} \int_{-\pi}^{\pi}\left\{\frac{k_{i} \cos \beta_{R}^{n_{i}}}{4 \pi \sinh k_{i}} \cdot \exp \left(\frac{\mathrm{j} 2 \pi D_{\mathrm{SR}_{i}}}{\lambda}\right)\right. \\
& \cdot \exp \left(\mathrm{j} 2 \pi \tau f_{\max } \cos \left(\alpha_{R}^{n_{i}}-\gamma_{R}\right) \cos \beta_{R}^{n_{i}}\right) \\
& \left.\cdot \exp \left[k_{i}\left(\cos \beta_{R}^{n_{i}} \cos \beta_{R 0}^{n_{i}} \cos \left(\alpha_{R}^{n_{i}}-\alpha_{R 0}^{n_{i}}\right)+\sin \beta_{R}^{n_{i}} \sin \beta_{R 0}^{n_{i}}\right)\right]\right\} d \alpha_{R}^{n_{i}} d \beta_{R}^{n_{i}}
\end{aligned}
$$

where $D_{\mathrm{SR}_{1}}=\delta_{T}\left[R_{S} \sin \beta_{R}^{n_{1}} \sin \varphi_{T}+Q_{n_{1}} \cos \varphi_{T} \cos \left(\theta_{T}-\alpha_{T}^{n_{1}}\right)\right] / \xi_{T_{1}}+\delta_{R}\left[\sin \beta_{R}^{n_{1}} \sin \varphi_{R}\right.$ $\left.+\cos \beta_{R}^{n_{1}} \cos \varphi_{R} \cos \left(\theta_{R}-\alpha_{R}^{n_{1}}\right)\right], D_{\mathrm{SR}_{2}}=\delta_{R}\left[\sin \beta_{R}^{n_{2}} \sin \varphi_{R}+\cos \beta_{R}^{n_{2}} \cos \varphi_{R} \cos \left(\theta_{R}-\alpha_{R}^{n_{2}}\right)\right]$ $+\delta_{T}\left[\xi_{R n_{2}} \sin \beta_{R}^{n_{2}} \sin \varphi_{T}+Q_{n_{2}} \cos \varphi_{T} \cos \left(\theta_{T}-\alpha_{T}^{n_{2}}\right)\right] / \xi_{T n_{2}}, Q_{n_{1}}=D+R_{S} \cos \beta_{R}^{n_{1}} \cos \alpha_{R}^{n_{1}}$, $Q_{n_{2}}=\left(\xi_{T n_{2}}^{2}-\xi_{R n_{2}}^{2} \sin ^{2} \beta_{R}^{n_{2}}\right)^{1 / 2} \quad, \quad \xi_{T n_{1}}=\left[\left(D+R_{S} \cos \beta_{R}^{n_{1}} \cos \alpha_{R}^{n_{1}}\right)^{2}+\left(R_{S} \sin \beta_{R}^{n_{1}}\right)^{2}\right]^{1 / 2} \quad$, $\xi_{T n_{2}}=R_{C} / \sin \left[\arccos \left(\cos \beta_{T}^{n_{2}} \cos \alpha_{T}^{n_{2}}\right)\right], \xi_{R n_{2}}=R_{C} / \sin \left[\arccos \left(\cos \beta_{R}^{n_{2}} \cos \left(\pi-\alpha_{R}^{n_{2}}\right)\right)\right]$. Due to $D>R_{S}$, and considering the relationship between the angles of the Tx and $\mathrm{Rx}$, we have the following approximations such as $\alpha_{T}^{n_{1}} \approx \beta_{T}^{n_{1}} \approx 0, \xi_{T n_{1}} \approx Q_{n_{1}} \approx D$ and $\xi_{T n_{2}} \approx \xi_{R n_{2}}$. Thus, $D_{\mathrm{SR}_{1}} \approx \delta_{R}\left[\sin \beta_{R}^{n_{1}} \sin \varphi_{R}+\cos \beta_{R}^{n_{1}} \cos \varphi_{R} \cos \left(\theta_{R}-\alpha_{R}^{n_{1}}\right)\right]+\delta_{T}\left[R_{S} \sin \beta_{R}^{n_{1}} \sin \varphi_{T} / D+\cos \varphi_{T} \cos \theta_{T}\right]$, $D_{\mathrm{SR}_{2}} \approx \delta_{R}\left[\cos \beta_{R}^{n_{2}} \cos \varphi_{R} \cos \left(\theta_{R}-\alpha_{R}^{n_{2}}\right)+\sin \beta_{R}^{n_{2}} \sin \varphi_{R}\right]+\delta_{T}\left[\sin \beta_{R}^{n_{2}} \sin \varphi_{T}\right.$ $\left.+\cos \beta_{R}^{n_{2}} \cos \varphi_{T} \cos \left(\theta_{T}-\alpha_{T}^{n_{2}}\right)\right]$.

\section{3) The STCF of DR component}

The STCF of DR component is expressed as

$$
\begin{aligned}
\rho_{p q, p^{\prime} q^{\prime}}^{\mathrm{DR}}\left(\delta_{T}, \delta_{R}, \tau\right)= & \eta_{\mathrm{DR}} \int_{-\pi}^{\pi} \int_{-\pi}^{\pi} \int_{-\pi}^{\pi} \int_{-\pi}^{\pi}\left\{\frac{k_{1} k_{2} \cos \beta_{R}^{n_{1}} \cos \beta_{T}^{n_{2}}}{16 \pi^{2} \sinh k_{1} \sinh k_{2}} \cdot \exp \left(\frac{\mathrm{j} 2 \pi}{\lambda} D_{\mathrm{DR}}\right)\right. \\
& \cdot \exp \left[k_{2}\left(\cos \beta_{T}^{n_{2}} \cos \beta_{T 0}^{n_{2}} \cos \left(\alpha_{T}^{n_{2}}-\alpha_{T 0}^{n_{2}}\right)+\sin \beta_{T}^{n_{2}} \sin \beta_{T 0}^{n_{2}}\right)\right] \\
& \cdot \exp \left[k_{1}\left(\cos \beta_{R}^{n_{1}} \cos \beta_{R 0}^{n_{1}} \cos \left(\alpha_{R}^{n_{1}}-\alpha_{R 0}^{n_{1}}\right)+\sin \beta_{R}^{n_{1}} \sin \beta_{R 0}^{n_{1}}\right)\right] \\
& \left.\cdot \exp \left[\mathrm{j} 2 \pi \tau f_{\max } \cos \left(\alpha_{R}^{n_{2}}-\gamma_{R}\right) \cos \beta_{R}^{n_{2}}\right]\right\} d \alpha_{T}^{n_{2}} d \beta_{T}^{n_{2}} d \alpha_{R}^{n_{1}} d \beta_{R}^{n_{1}}
\end{aligned}
$$

where $D_{\mathrm{DR}}=\delta_{T}\left[\xi_{R n_{2}} \sin \beta_{R}^{n_{2}} \sin \varphi_{T}+Q_{n_{2}} \cos \varphi_{T} \cos \left(\theta_{T}-\alpha_{T}^{n_{2}}\right)\right] / \xi_{T n_{2}}+\delta_{R}\left[\sin \beta_{R}^{n_{1}} \sin \varphi_{R}\right.$ $\left.+\cos \beta_{R}^{n_{1}} \cos \varphi_{R} \cos \left(\theta_{R}-\alpha_{R}^{n_{1}}\right)\right]$.

\subsection{Amplitude and Phase PDFs}

The amplitude and phase of the complex fading envelope $h_{p q}(t)$ are $z(t)=\left|h_{p q}(t)\right|$ and 
$\theta(t)=\arg \left\{h_{p q}(t)\right\}$, respectively. Hence, according to the similar computation procedure offered in [24], the amplitude PDF of the 3D GBSM can be derived as

$$
\begin{aligned}
g_{z}(z) & =4 \pi^{2} z \int_{0}^{\infty}\left[\prod_{n_{1}=1}^{N_{1}} J_{0}\left(2 \pi\left|Q_{\mathrm{SR}_{1}}\right| x\right) \cdot \prod_{n_{2}=1}^{N_{2}} J_{0}\left(2 \pi\left|Q_{\mathrm{SR}_{2}}\right| x\right) \cdot \prod_{n_{1}, n_{2}=1}^{N_{1}, N_{2}} J_{0}\left(2 \pi\left|Q_{\mathrm{DR}}\right| x\right)\right] \\
& \cdot J_{0}(2 \pi z x) \cdot J_{0}\left(2 \pi K_{0} x\right) x \mathrm{~d} x
\end{aligned}
$$

and the phase PDF can be similarly expressed as

$$
\begin{aligned}
g_{\theta}(\theta)= & 2 \pi \int_{0}^{\infty} \int_{0}^{\infty}\left[\prod_{n_{1}=1}^{N_{1}} J_{0}\left(2 \pi\left|Q_{\mathrm{SR}_{1}}\right| x\right) \cdot \prod_{n_{2}=1}^{N_{2}} J_{0}\left(2 \pi\left|Q_{\mathrm{SR}_{2}}\right| x\right) \cdot \prod_{n_{1}, n_{2}=1}^{N_{1}, N_{2}} J_{0}\left(2 \pi\left|Q_{\mathrm{DR}}\right| x\right)\right] \\
& \cdot J_{0}\left(2 \pi x \sqrt{z^{2}+K_{0}^{2}-2 z K_{0} \cos \left(\theta-\theta_{r e}\right)}\right) x z \mathrm{~d} x \mathrm{~d} z
\end{aligned}
$$

where $\quad Q_{\mathrm{SR}_{i}}=\sqrt{\frac{\eta_{\mathrm{SR}_{i}}}{N_{i}(K+1)}}, \quad Q_{\mathrm{DR}}=\sqrt{\frac{\eta_{\mathrm{DR}}}{N_{1} N_{2}(K+1)}} \quad(\quad i=1,2 \quad), \quad K_{0}=\sqrt{\frac{K}{K+1}} \quad$, $\theta_{r e}=\arg \left\{h_{p q}^{\mathrm{LOS}}(t)\right\}$ and $J_{0}(\cdot)$ represents the zeroth-order Bessel function of the first kind.

\subsection{Channel Capacity}

In this section, the MIMO channel capacity is derived. Channel capacity refers to the maximum average information transmitting rate of a channel. Furthermore, explicit MIMO system structure and theory are proposed in literature [25] and the authors proved mathematically that channel capacity can be increased by increasing the transmitting and receiving antennas without augmenting the bandwidth. Under an average transmit power constraint, the instantaneous channel capacity of a stochastic massive MIMO channel can be defined as [26]

$$
C(t)=\log _{2}\left[\operatorname{det}\left(\mathbf{I}_{\min }+\frac{\rho_{S N R}}{M_{T}} \mathbf{H}(t) \mathbf{H}^{\mathrm{H}}(t)\right)\right]
$$

where $\rho_{S N R}$ is the average signal-to-noise ratio(SNR) at the $\mathrm{Tx}, \mathbf{I}_{\min }$ represents the $M_{R} \times M_{R}$ identity matrix, $(\cdot)^{\mathrm{H}}$ denotes the transpose conjugate operation and $\operatorname{det}(\cdot)$ designates the matrix determinant. Here, it's assumed that $M_{R} \leq M_{T}$ which is the condition that space-division multiplexing must meet [27].

In general, channel capacity has two significant definitions which are ergodic capacity and outage capacity. Furthermore, the ergodic capacity is the mathematic expectation of the instantaneous capacity over time and is defined as[27]

$$
C_{\text {mean }}=\mathbf{E}[C(t)]=\mathbf{E}\left\{\log _{2}\left[\operatorname{det}\left(\mathbf{I}_{\text {min }}+\frac{\rho_{S N R}}{M_{T}} \mathbf{H}(t) \mathbf{H}^{\mathrm{H}}(t)\right)\right]\right\}
$$

and the outage capacity $C_{\text {out }}$ means the most suitable capacity under the condition that message is correctly received. The $C_{\text {out }}$ is related to an outage probability $P_{\text {out }}$ which satisfies 


$$
\begin{aligned}
P_{\text {out }} & =p\left(\mathbf{H}: C(t)<C_{\text {out }}\right) \\
& =p\left(\mathbf{H}: \log _{2}\left[\operatorname{det}\left(\mathbf{I}_{\min }+\frac{\rho_{S N R}}{M_{T}} \mathbf{H}(t) \mathbf{H}^{\mathrm{H}}(t)\right)\right]<C_{\text {out }}\right)
\end{aligned}
$$

where $p(\cdot)$ is a probability function.

\section{Simulation Results and Analysis}

The statistical properties of the proposed 3D GBSM are investigated in this section. Referring to the literature [28-29] and the 3rd Generation Partnership Project (3GPP) protocol [30], the simulation parameters chosen are: $f_{c}=2 \mathrm{GHz}, D=500 \mathrm{~m}, R_{C}=R_{S}=4 \mathrm{~m}, f_{\max }=500 \mathrm{~Hz}, \gamma_{R}=0^{\circ}$, $\theta_{T}=\theta_{R}=45^{\circ}, \eta_{\mathrm{SR}_{1}}=0.125, \eta_{\mathrm{SR}_{2}}=0.325, \eta_{\mathrm{DR}}=0.55$. The environmental coefficient $k_{i}$ is related to the effective scatterer distribution. Specifically, for the denser environmental scatterers, the smaller value of $k_{i}$ is used. We set $k_{1}=4, k_{2}=15, M_{T}=128$ and $M_{R}=64$. In addition, when all elevation angles are set as $0^{\circ}$, the 3D GBSM will be translated into 2D model which can be taken as a contrast. The performances are compared with the 3D model, 2D model and the geometric elliptical scattering model proposed in [31].

\subsection{Temporal Auto-Correlation Function (ACF)}

The expression of the temporal ACF can be derived from STCF by setting $\delta_{T}=\delta_{R}=0$ and is expressed as

$$
\rho_{p q, p^{\prime} q^{\prime}}(\tau)=\rho_{p q, p^{\prime} q^{\prime}}(0,0, \tau)
$$

Fig. 2 shows the absolute values of the normalized temporal ACFs for the proposed 3D model, the 3D reference model and the 2D model. It is clear that the absolute values of ACFs decrease as the time separation increases whether in the 3D or 2D model which implies that auto-correlation values drop. Moreover, the ACF value of the 3D reference model is always lower than that of the 2D model but higher than that of the 3D proposed model.

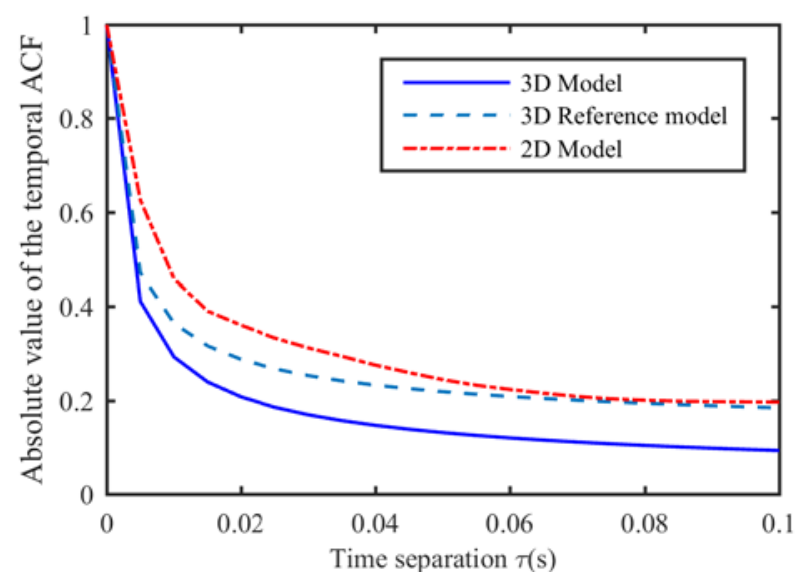

Fig. 2. The absolute values of the temporal ACFs for the 3D and 2D models $\left(\delta_{T}=\delta_{R}=0\right.$ ). 


\subsection{Spatial Cross-Correlation Function (CCF)}

The CCF can be used to describe the mutual influence between different antenna elements under the same time. In order to analyze the CCF, $\tau$ is set as 0 and $\delta_{T}$ is set as $0.5 \lambda$, thus, the spatial CCF can be expressed as

$$
\rho_{p q, p^{\prime} q^{\prime}}\left(\delta_{R}\right)=\rho_{p q, p^{\prime} q^{\prime}}\left(0.5 \lambda, \delta_{R}, 0\right)
$$

Fig. 3 presents the absolute values of the normalized spatial CCFs for the 3D model ( $\varphi_{T}=45^{\circ}, \varphi_{R}=45^{\circ}$ and $\varphi_{T}=45^{\circ}, \varphi_{R}=60^{\circ}$ ), the 3D reference model and the 2D model $\left(\varphi_{T}=\varphi_{R}=0^{\circ}\right.$ ). Similarly, it is clear that as the normalized spacing between antenna elements at the Rx increase, the absolute values of CCFs decrease, which signifies that the spatial correlation gradually diminishes. Furthermore, the CCF value of the 2D model is always higher than that of the 3D proposed model, which means the 2D model overestimates the spatial correlation. Moreover, Compared with the 3D reference model, the model in this paper has lower CCF. In addition, the elevation angle of the Rx antenna array $\varphi_{R}$ has significant impact on CCFs. Specifically, when $0 \leq \varphi_{R} \leq 90^{\circ}$, the bigger $\varphi_{R}$, the lower CCF values.

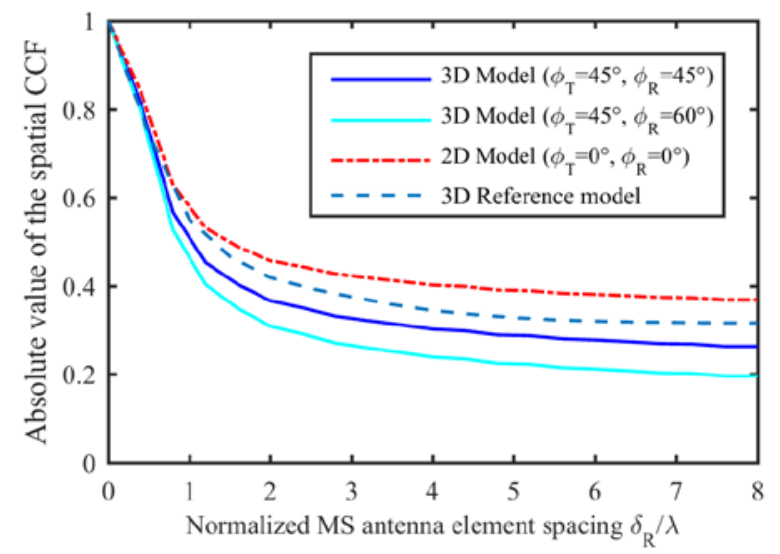

Fig. 3. The absolute values of the spatial CCFs for the 3D and 2D models $\left(\tau=0, \delta_{T} / \lambda=0.5\right)$.

\subsection{Amplitude and Phase PDFs}

Figs. 4 and 5 show that the Rician factor $K$ has a significant influence on the amplitude and phase PDFs. Specifically, the curve of the amplitude PDF shifts to the right and the peak value decreases as $K$ increases. As to the phase PDF, it is clear that increasing the $K$ will improve the peak value however, the mean value remain constant. 


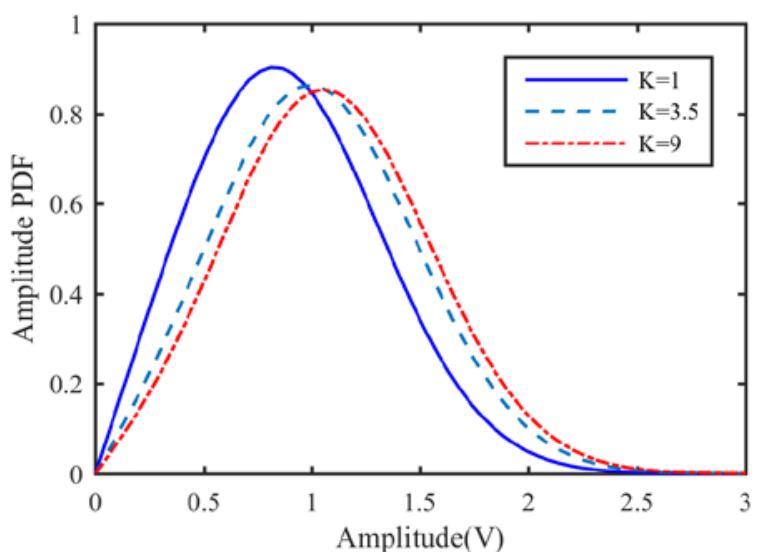

Fig. 4. The amplitude PDFs with $K=1, K=3.5$ and $K=9$.

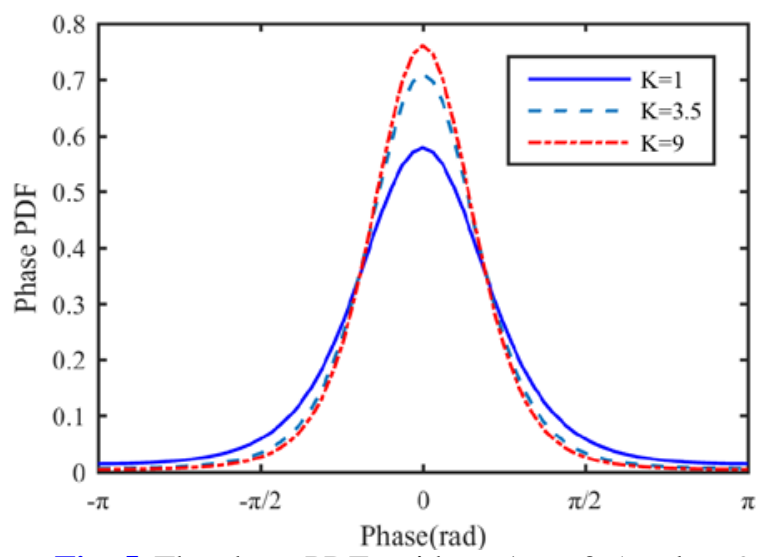

Fig. 5. The phase PDFs with $K=1, K=3.5$ and $K=9$.

\subsection{Channel Capacity Analysis}

The factors effecting the channel capacity such as SNR and space-time correlation are investigated in this section. Fig. 6 shows the ergodic capacity against $\rho_{S N R}$ with $\delta_{T} / \lambda=\delta_{R} / \lambda=5$ for both 3D and 2D models. As expected, the ergodic capacity increases as $\rho_{S N R}$ increases however, the growth rate gradually decreases. Furthermore, similar to the previous temporal and spatial correlations, the 3D model always has a higher ergodic capacity than the 2D model. Fig. 7 presents the ergodic capacity as a function of the normalized antenna element spacing at the Tx and Rx with $\rho_{S N R}=10 \mathrm{~dB}$ for the 3D model. It is observed that when $\delta_{T} / \lambda<3$, increasing the normalized antenna element spacing $\delta_{T} / \lambda$ increases the ergodic capacity. However, it is clear that increasing $\delta_{T} / \lambda$ has a negligible effect on the ergodic capacity when $\delta_{T} / \lambda>3$. In addition, the ergodic capacity can be improved by incrasing $\delta_{R} / \lambda$ but cannot be increased indefinitely. 


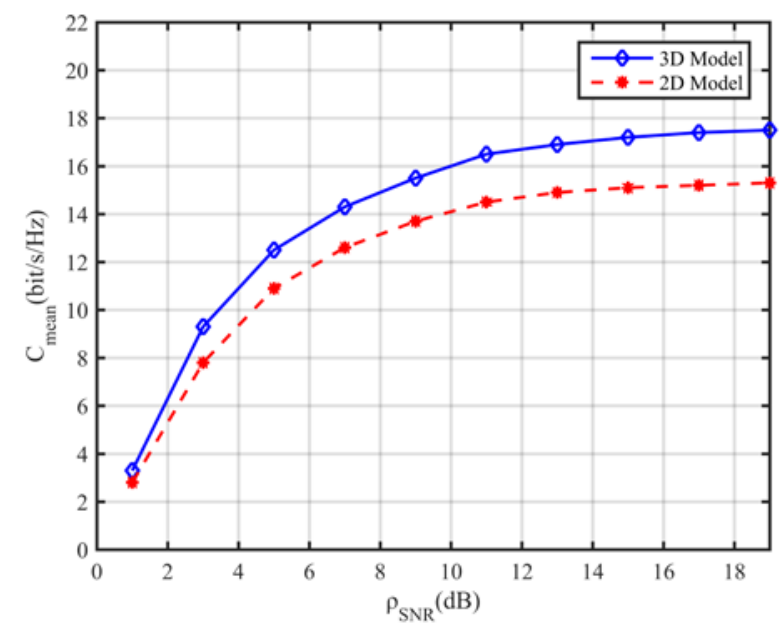

Fig. 6. The ergodic capacity against $\rho_{S N R}$ for the 3D and 2D models.

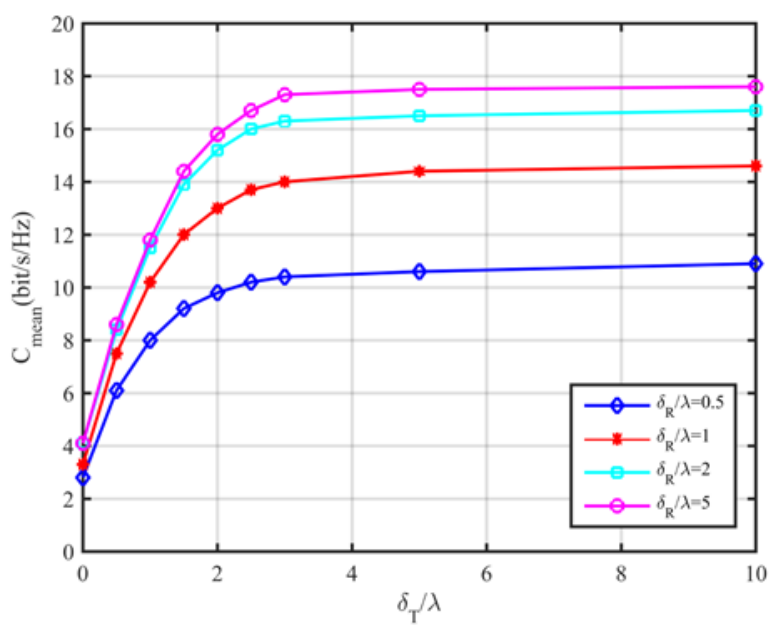

Fig. 7. The ergodic capacity as a function of the normalized antenna element spacing at the Tx and Rx for the 3D model

\section{Conclusion}

In this paper, a neoteric 3D "cylinder-sphere" theoretical geometrical model for the non-isotropic scattering massive MIMO channels in tunnels is proposed. Furthermore, the matrix of complex fading envelopes for the massive MIMO channel is derived. Then, the STCF, amplitude and phase PDFs are investigated and the derived channel capacity is analyzed by numerical simulations. The statistics of the 3D and 2D models are also verified by simulations. It is obvious that the 3D model has a lower space-time correlation compared with the 2D model. Besides, the time separation and the normalized antenna element spacing at the Rx both have negative effect on the STCF and the Rician factor $K$ has a significant impact on the amplitude and phase PDFs. Furthermore, numerical simulations have shown that the channel capacity increases as the SNR and normalized antenna element spacing increase, respectively. In a nutshell, the proposed 3D massive MIMO GBSM can characterize the channel environment in tunnels more practically than the traditional 2D models. The presented 
research work can be taken as a reference to establish the future communication systems operating in subway tunnels.

\section{References}

[1] T. L. Marzetta, "Noncooperative cellular wireless with unlimited numbers of base station antennas," IEEE Transactions on Wireless Communications, vol. 9, no. 11, pp. 3590-3600, November, 2010. Article (CrossRef Link).

[2] D. C. Araújo, T. Maksymyuk, A. L. F. de Almeida, T. Maciel, J. C. M. Mota and M. Jo, "Massive MIMO: survey and future research topics," IET Communications, vol. 10, no. 15, pp. 1938-1946, October, 2016. Article (CrossRef Link).

[3] X. Ge, R. Zi, H. Wang, J. Zhang and M. Jo, "Multi-user massive MIMO communication systems based on irregular antenna arrays," IEEE Transactions on Wireless Communications, vol. 15, no. 8, pp. 5287-5301, August, 2016. Article (CrossRef Link).

[4] E. G. Larsson, O. Edfors, F. Tufvesson and T. L. Marzetta, "Massive MIMO for next generation wireless systems," IEEE Communications Magazine, vol. 52, no. 2, pp. 186-195, February, 2014. Article (CrossRef Link).

[5] J. Yao, H. Ren and Q. Liu, "Massive MIMO channel modeling using map-based ray tracing method," in Proc. of 7th IEEE International Symposium on Microwave, Antenna, Propagation, and EMC Technologies, pp. 1-5, October 24-27, 2017. Article (CrossRef Link).

[6] H. W. Son, J. W. Yoo, G. J. Jin and B. Jang, "A fast 3-D ray tracing method for wave propagation prediction using a reflection tube tree," in Proc. of International Symposium on Antennas and Propagation, pp. 1-2, October 30-November 2, 2017. Article (CrossRef Link).

[7] Y. Yuan, C. Wang, Y. He, M. M. Alwakeel and e. M. Aggoune, "3D wideband non-stationary geometry-based stochastic models for non-isotropic MIMO vehicle-to-vehicle channels," IEEE Transactions on Wireless Communications, vol. 14, no. 12, pp. 6883-6895, December, 2015. Article (CrossRef Link).

[8] M. Boutin, A. Benzakour, C. L. Despins and S. Affes, "Radio wave characterization and modeling in underground mine tunnels," IEEE Transactions on Antennas and Propagation, vol. 56, no. 2, pp. 540-549, February, 2008. Article (CrossRef Link).

[9] R. Verma, S. Mahajan and V. Rohila, "Classification of MIMO channel models," in Proc. of 16th IEEE International Conference on Networks, Sensing and Control, pp. 1-4, April 6-8, 2008. Article (CrossRef Link).

[10] J. Zhao, S. Wang, X. Liu and Y. Gong, "Geometry-based stochastic modeling for non-stationary high-speed train MIMO channels," in Proc. of IEEE 84th Vehicular Technology Conference (VTC-Fall), pp. 1-5, September 18-21, 2016. Article (CrossRef Link).

[11] J. Y. Yan, B. Ai, K. Guan, D. P. He, X. Lin, B. Hui and J. H. Kim, A. Hrovat "A geometry-based stochastic channel model for the millimeter-wave band in a 3GPP high-speed train scenario," IEEE Transactions on Vehicular Technology, vol. 67, no. 5, pp. 3853-3865, May, 2018. Article (CrossRef Link).

[12] X. Cheng, C. X. Wang, Y. Yuan, D. I. Laurenson, S. Salous, and A. V. Vasilakos, "An adaptive geometry-based stochastic model for non-isotropic MIMO mobile-to-mobile channels," IEEE Transactions on Wireless Communications, vol. 8, no. 9, pp. 4824-4835, September, 2009. Article (CrossRef Link).

[13] S. Yoo, J. Lee and K. Kim, "Geometry-based one-ring models for MIMO systems: modeling accuracy assessment and improvement," IEEE Transactions on Wireless Communications, vol. 15, no. 7, pp. 4583-4597, July, 2016. Article (CrossRef Link).

[14] 3GPP, "Technical specification group radio access network study on spatial channel model for multiple input multiple output (MIMO) simulations (Release 9),” 3GPP TR 25.996, V9.0.0, Tech. Rep., 2009. 
[15] H. D. Zheng and X. Y. Nie, "GBSR model for MIMO channel and its space-time correlation analysis in tunnel," in Proc. of IEEE International Symposium on Knowledge Acquisition and Modeling Workshop, pp. 221-224, December 21-22, 2008. Article (CrossRef Link).

[16] J. Chen, S. Wu, S. Liu, C. Wang and W. Wang, "On the 3-D MIMO channel model based on regular-shaped geometry-based stochastic model," in Proc. of 2015 IEEE International Symposium on Antennas and Propagation, pp. 1-4, November 9-12, 2015. Article (CrossRef Link).

[17] R. He, G. L. Stüber, B. Ai and Z. Zhong, “A two-cylinder based polarized MIMO channel model," in Proc. of 2017 General Assembly and Scientific Symposium of the International Union of Radio Science, pp. 1-4, August 19-26, 2017. Article (CrossRef Link).

[18] Y. Yuan, C. X. Wang, X. Cheng, B. Ai and D. I. Laurenson, "Novel 3D geometry-based stochastic models for non-isotropic MIMO vehicle-to-vehicle channels,” IEEE Transactions on Wireless Communications, vol. 13, no. 1, pp. 298-309, January, 2014. Article (CrossRef Link).

[19] F. Wang, X. Cheng, L. Yang, B. Yu and B. Jiao, "On the achievable capacity of dual-polarized antenna systems in 3D indoor scenarios,” in Proc. of 2013 IEEE/CIC International Conference on Communications in China, pp. 605-610, August 12-14, 2013. Article (CrossRef Link).

[20] N. I. Fisher, T. Lewis and B. J. J. Embleton, "Statistical Analysis of Spherical Data,” Cambridge University Press, Cambridge, U.K., 1987. Article (CrossRef Link).

[21] Y. Liu, B. Ai, K. Guan and B. Chen, "Antenna array configurations for 3D MIMO system in high speed railway scenario," in Proc. of 2015 IEEE International Symposium on Antennas and Propagation \& USNC/URSI National Radio Science Meeting, pp. 719-720, July 19-24, 2015. Article (CrossRef Link).

[22] C. A. Gutiérrez, J. T. Gutiérrez-Mena, J. M. Luna-Rivera, D. U. Campos-Delgado, R. Velázquez and M. Pätzold, "Geometry-based statistical modeling of non-WSSUS mobile-to-mobile rayleigh fading channels," IEEE Transactions on Vehicular Technology, vol. 67, no. 1, pp. 362-377, January, 2018. Article (CrossRef Link).

[23] X. Cheng, C. X. Wang, Y. Yuan, D. I. Laurenson and X. Ge, "A novel 3D regular-shaped geometry-based stochastic model for non-isotropic MIMO mobile-to-mobile channels,” in Proc. of 2010 IEEE 72nd Vehicular Technology Conference - Fall, pp. 1-5, September 6-9, 2010. Article (CrossRef Link).

[24] M. Patzold and B. Talh, "On the statistical properties of sum-of-cisoids-based mobile radio channel models,” in Proc. of 10th International Symposium on Wireless Personal Multimedia Communications, pp. 394-400, December 8-11, 2007. Article (CrossRef Link).

[25] J. M. Molina-Garcia-Pardo, M. Lienard, P. Degauque, E. Simon and L. Juan-Llacer, “On MIMO channel capacity in tunnels,” IEEE Transactions on Antennas and Propagation, vol. 57, no. 11, pp. 3697-3701, November, 2009. Article (CrossRef Link).

[26] Y. T. Pan, G. X. Zheng and T. Wang, "Investigation of MIMO channel correlation and capacity in curved subway tunnel,” IEEE Antennas and Wireless Propagation Letters, vol. 15, no. 4, pp. 1698-1702, February, 2016. Article (CrossRef Link).

[27] A. G. Zajic and G. L. Stuber, "Three-dimensional modeling, simulation, and capacity analysis of space-time correlated mobile-to-mobile channels," IEEE Transactions on Vehicular Technology, vol. 57, no. 4, pp. 2042-2054, July, 2008. Article (CrossRef Link).

[28] Y. Zhang, Y. Liu, J. Sun, C. X. Wang and X. Ge, "Impact of different parameters on channel characteristics in a high-speed train ray tracing tunnel channel model," in Proc. of 2017 IEEE 85th Vehicular Technology Conference (VTC Spring), pp. 1-5, June 4-7, 2017. Article (CrossRef Link).

[29] Guillermo Acosta-Marum and M. A. Ingram, "Six time- and frequency- selective empirical channel models for vehicular wireless LANs,” IEEE Vehicular Technology Magazine, vol. 2, no. 4, pp. 4-11, December, 2007. Article (CrossRef Link).

[30] 3GPP, “Technical specification group radio access network study on 3D channel model for LTE (Release 12),” 3GPP TR 36.873 V12.2.0, Tech. Rep., 2015. Article (CrossRef Link).

[31] M. Patzold and B. O. Hogstad, "A wideband MIMO channel model derived from the geometric elliptical scattering model," in Proc. of 2006 3rd International Symposium on Wireless Communication Systems, pp. 138-143, September 6-8, 2006. Article (CrossRef Link). 


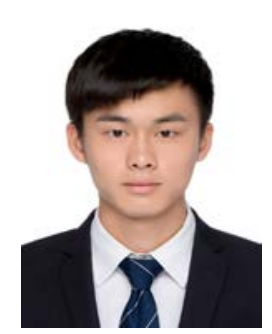

Yukang Jiang: He was born in Chizhou, Anhui, China in 1994. He received his BSc degree with distinction in Communication Engineering from Tongji University, Shanghai, China, in 2017. He has been a Master student at School of Electronic and Information Engineering, Tongji University since 2017. His main research interests include the researchh and application of massive MIMO technology and wireless channel modeling.

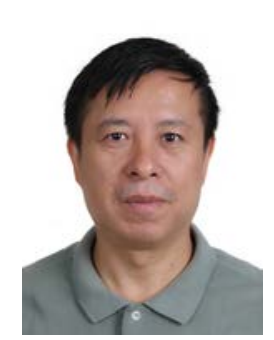

Aihuang Guo: He is now working at Tongji University as a full professor and Ph. D. candidate advisor. He is engaged in the research and teaching of broadband wireless communications networks, signal and information processing. He has authored 2 books and published over 100 academic research papers in his research area. He has presided over the sub-topics of the national science and technology support program, the National Natural Science Foundation of China and the open topics of State Key Laboratory.

Prof. Aihuang Guo is a director of China Education Society of Electronics. He is also a peer appraisal expert of the National Natural Science Foundation Committee. He has been working as a professional reviewer for many academic journals such as ACTA Electronica Sinica, Journal of Electronics \& Information Technology and Systems Engineering \& Electronics. His research interests include the research and application of massive MIMO technology, wireless communication systems and channel modeling.

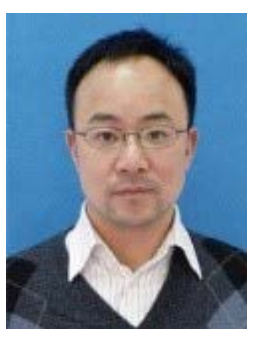

Jinbai Zou: He received his BSc degree with distinction in Wireless Communication from Beijing Jiaotong University, Beijing, China, in 1994. He received his Master degree from Tongji University in 2006. He has been a Ph. D. student at Tongji University since 2014. He is a professor of engineering. He has hold 4 invention patents and published 3 scientific papers in his area. His main research interests include Mobile communication technology, rail transit transmission technology and rail transit dispatch communication technology.

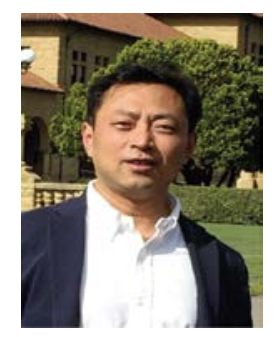

Bo Ai: He received his Master and Ph. D. degrees from Xidian University, Shanxi, China. He graduated from Tsinghua University as an excellent postdoctoral research fellow in 2007. He is now working at Beijing Jiaotong University as a professor and Ph. D. advisor. He is a vice director of State Key Laboratory of Rail Traffic Control and Safety and also a vice director of Modern Communications Research Institute. $\mathrm{He}$ has authored/co-authored/translate 10 books and published over 150 academic research papers in his research area. He has applied for 26 invention patents. He has been the team leader for 26 national research projects and has won some excellent scientific research prizes.

Prof. Bo Ai is an IEEE Senior Member and an IET Fellow. He is an associate Editor of IEEE Transactions on Consumer Electronics and an Editorial Committee Member of the Wireless Personal Communications journal. He is also the Lead Guest Editor of International Journal of Antennas and Propagations. His research interests include radio wave propagation, wireless channel measurement and channel modeling, and mobile communications for rail traffic systems. 